\title{
Die DEGUM-Verwaltung stellt sich neu auf
}

Liebe Kolleginnen und Kollegen, liebe DEGUM-Mitglieder,

Sie werden die Veränderungen wahrscheinlich bereits bemerkt haben, sei es beim Telefonat mit einer neuen Mitarbeiterin oder beim Suchen einer Kontaktadresse auf unserer Homepage.

Seit 1. April 2014 sitzt die Geschäftsstelle der DEGUM in Berlin, in unmittelbarer Nachbarschaft der Ultraschall-Akademie. In Verbindung mit der schrittweisen Reduktion der Präsenz in Bonn ist dies aber nur das äußere Zeichen einer strukturellen Neuausrichtung der Verwaltung der DEGUM. So werden zukünftig sowohl die Öffentlichkeitsarbeit des (erweiterten)
Vorstands als auch die wissenschaftlichen Aktivitäten der Gesellschaft von Mitarbeitern der dazu in Berlin neu eingerichteten Referate organisiert und koordiniert. Das beinhaltet die Präsenz in bzw. bei medizinischen und wissenschaftlichen Gremien, Verbänden und Fachgesellschaften sowie die Begleitung und Initiierung wissenschaftlicher Projekte und Studien.

Bereits seit Mitte 2013 befindet sich das Schatzmeisteramt in Berlin, die Mitgliederverwaltung wird, parallel mit der Umsetzung der EDV-Hardware von Bonn nach Berlin, in Kürze folgen. Spätestens im Herbst 2014, wenn das Zertifizierungsverfahren im Rahmen des Mehrstufenkonzepts Internet-basiert abgewickelt werden kann, wird auch dieser interne Verwaltungsbereich komplett in Berlin angesiedelt sein.

Verbleiben sollen im Bonner Büro die Koordination des Vorstands, die internationale Vertretung der DEGUM gegenüber EFSUMB, WFUMB und Partnergesellschaften wie ÖGUM und SGUM, und der Service zu Fragen rund um das DEGUMKurssystem.

Ziele dieser bereits vor einiger Zeit vom (erweiterten) Vorstand beschlossenen und jetzt eingeleiteten Maßnahmen sind die Steigerung der „Wahrnehmung“ der DEGUM innerhalb der medizinischen wissenschaftlichen „Landschaft“ in Deutschland sowie die Professionalisierung des Service-Angebots für Sie als DEGUM-Mitglied.

MG, Sekretär 